\title{
Odyssey of Human Creative Genius: From Astronomical Heritage to Space Technology Heritage
}

\author{
Olga Dluzhnevskaya ${ }^{1}$ and Mikhail Marov ${ }^{2}$ \\ ${ }^{1}$ Institute for Astronomy, Russian Academy of Sciences, \\ National Focal Point for the Russian Federation, Astronomy and World Heritage Initiative \\ email: olgad@inasan.ru \\ ${ }^{2}$ Vernadsky Institute, Russian Academy of Sciences, \\ Nominated Chair of WG on Space Technology, UNESCO WH Committee \\ email: marovmail@yandex.ru
}

\section{Introduction and Historical Highlights}

Astronomy was one of the most important sciences in the ancient world. It was rooted in naked eye observations and primitive stone instruments for astrometric measurements to determine the positions of the Sun, Moon, planets and some stars that had both practical and sacred meaning. That is why the majority of archaeoastronomical monuments are simultaneously observatories and sanctuaries, with burials and altars.

Interest in the investigation of ancient monuments as instruments for astronomical observations has grown significantly in recent decades. Since 1981, the "Oxford" international conferences on archaeoastronomy have been held every 3-5 years, organized since 1995 by the International Society for Archaeoastronomy and Astronomy in Culture (ISAAC) which was founded in the USA in that year. The European Society for Astronomy in Culture (SEAC) also deals with the problems of archaeoastronomy and holds scientific conferences annually. In Russia, the first conference on archaeoastronomy was held in 1996 in Moscow, followed by the SEAC conference "Astronomy of ancient civilizations" during the JENAM meeting in 2000, and the International symposium on "Astronomy 2005-modern state and prospects" in 2005.

A new milestone started with the UNESCO Initiative on "Astronomy and World Heritage". Throughout the years, UNESCO has been working hard to preserve humankind's achievements, astronomy included, as World Heritage. The breathtaking monuments of ancients civilizations such as the Decorated Grottoes of The Vézère Valley (France), Stonehenge (Great Britain), the Lines of Nasca (Peru), the Pyramids of Giza (Egypt), the Temple of Heaven (China), and Ulugh Beg's Observatory in Samarqand (Uzbekistan), to mention a few, have been recognized. These also bear an invaluable educational mission.

In 2003, the UNESCO World Heritage Centre (WHC) set up a new project to reveal and preserve the objects of archaeoastronomy of historical and cultural value all over the world. The First International Meeting of experts "Archaeoastronomical objects and observatories", organized by the WHC and the Regional European Bureau on Science, was held in 2004 in Venice, Italy. The strategy of the thematic program "Astronomy and World Heritage" and the general criteria for the selection of archaeoastronomical sites/observatories were considered, including (i) Objects situated or related to celestial objects or astronomical events; (ii) Images of the sky and/or of celestial objects and 
astronomical events; (iii) Observatories and instruments; and (iv) Objects closely connected with the history of astronomy.

\section{Space Exploration: Baseline and Venue}

Space exploration manifested a new great milestone in the development of human civilization. It made possible observations in every wavelength with incremental precision and, also, direct in situ measurements on other worlds. The Astronomy and World Heritage Initiative should encompass various facilities related to space exploration. Basically, great breakthroughs of modern astronomy were achieved thanks to space-born instruments and planetary space missions. Indeed, space astronomy ensured very significant progress in astrophysics, gaining invaluable knowledge about space objects and the Universe as a whole, thus broadening human horizons tremendously. It is therefore essential to include heritage related to Space Astronomy as an important segment of astronomical World Heritage.

The project on technological heritage connected with space exploration arises as a logical extension of the Astronomy and World Heritage Initiative because it is intrinsically related with the most important breakthroughs in space science and additionally, it is rooted in space technology. The idea was put forward at the Astronomy and World Heritage meeting held in Kazan, Russia in August, 2009 (ASTROKAZAN 2009), subject to further discussions and clarifications. A first step towards the goal has been undertaken in the contributions by David DeVorkin and Mikhail Marov on Space Achievements as World Heritage that form Ch. 15 of the first ICOMOS-IAU Thematic Study on the Heritage Sites of Astronomy and Archaeoastronomy (Ruggles \& Cotte (2010)). It is generally understood that the proposed segment on Space Astronomy/Technology World Heritage should have an international significance in terms of human beings' tight relationships with the sky. Obviously, an international team of specialists should be further involved in the process of collecting respective materials/documentation and writing proposals to accommodate the World Heritage Convention.

A synopsis of the most important events along the way is as follows:

- Round-table discussion in the Russian Academy of Sciences aiming to define a core of the Thematic Initiative on the heritage of science and technology (2005);

- 50th anniversary of the Sputnik: "Fenêtres sur le Cosmos: Spoutnik et l'Aube de l'Age Spatial" organized at the French Senate by ESA/CNES, Paris, France;

- Thematic research proposal "Odyssey of human creative genius: towards the protection of space technological heritage connected with space exploration" (2007);

- International Conference on "Astronomy and World Heritage: Across Time and Continents", Kazan, Republic of Tatarstan, Russia (2009);

- ICOMOS/IAU Thematic Study on the Heritage of Astronomy, Cairo, Egypt (2010);

- International Seminar on the Heritage of Astronomy in the Institute of Astrophysics, Paris, France (2011); and

- UNESCO World Heritage Committee meeting in St. Petersburg, Russia (2012).

At the Kazan conference, a preliminary definition was given for the first time of types of technological sites and facilities connected with space exploration, and it was proposed how to identify the clearly defined landscape designed and created intentionally by man and intrinsically associated with the Launch Pad and the development of related structures. The focus was upon the most prominent parts of space networks specially designed and built for manned flights and the historical sites where the concepts of spaceflight were pioneered and original space vehicle designs were tested. At the Cairo 
meeting, space heritage was additionally specified as heritage related to the process of carrying out science in space, heritage related to manned space flight/exploration, and human cultural heritage that remains off the surface of planet Earth. In turn, at the Paris meeting the tentative proposal of fixed sites and facilities pertaining specifically to space astronomy and/or generally to space science - in particular, ground space facilities and launch pads (cosmodromes) — was discussed.

The UNESCO World Heritage Committee in St. Petersburg in 2012 preliminarily endorsed Space Astronomy as a segment of Astronomical Heritage and, basically, as a segment of Space Technology Heritage. It was recommended to set up an International Working Group under the UNESCO umbrella in order to discuss the main issue and develop proposals on how to progress the Space Technology Initiative and to accommodate the World Heritage Convention. In Decision 36COM5D adopted at the 36 th Session of the World Heritage Committee in 2012 it is stated: "The World Heritage Committee . . Also welcomes financial and technical support provided by States Parties and the International Astronomical Union for [the] Thematic Initiative 'Astronomy and World Heritage' since 2003 and also encourages cooperation between the UNESCO World Heritage Centre, specialized agencies and relevant interdisciplinary scientific initiatives towards the elaboration of a Global Thematic Study on [the] Heritage of Science and Technology, including studies and research on technological heritage connected with space exploration; [and] Further encourage States Parties, international organizations and other donors to contribute to the thematic programmes and initiatives and also requests an updated report on Thematic Programmes to the World Heritage Committee at its 38th session in $2014 "$.

Following this resolution, the Director of the WHC, Mr. Kishore Rao, in a letter to interested parties on June 30, 2012, emphasized the point that "In the light of this decision, the World Heritage Centre would like to identify all main actors concerned in order to enhance international cooperation and to define new partnerships. Two working groups were already created within the framework of this initiative - an International Working Group on Astronomy and World Heritage chaired by Prof. Clive Ruggles and a first expert Working Group on technological heritage connected with space exploration chaired by Prof. Mikhail Marov, Academician of the Russian Academy of Science." In addition, the UNESCO Associated Director-General for Culture, Mr. Francesco Bandarin, in his letter to Prof. Mikhail Marov dated July 20, 2012, underlined that "a number of decisions regarding development of innovative strategic approaches in the implementation of the World Heritage Convection were taken by the World Heritage Committee at the 36th session. Among them, the Committee's first decision on technological heritage connected with space exploration is indeed highly symbolic. I would like to underline the very active role the high-level Russian specialists play in the promotion and support of this new initiative ... I am confident that under your chairmanship and with the involvement of all specialized agencies, institutions and centres concerned, this study on technological heritage connected with space exploration will be the start to our common flagship activities."

It is worth noting that the International Astronomical Union has supported this new UNESCO Initiative since the very beginning. Quoting from the MOU adopted during the 28th IAU General Assembly (Beijing, China, August 24, 2012): "The IAU expresses its continuing support to the UNESCO Thematic Initiative 'Astronomy and World Heritage' and in response to the UNESCO promotion, is willing to further extend this Initiative over Space Science and Technology with the main focus placed on Space Astronomy and relevant facilities." 


\section{Space Astronomy and Space Technology: A Synergy}

There is a basic synergy between Space Astronomy and Space Technology, the latter serving as a driver to progress with astronomy. The Space Heritage Initiative can be further extended to many historically important achievements in space science and technology spin-offs. Robotic and manned flights aiming eventually to establish a permanent human habitat in space and to follow up by extending this through the Solar system serve as brilliant examples of Space Science and Technology Synergy. However, many important questions remain unsolved and are not yet clearly defined. The key question is how to recognize and/or commemorate space-related objects and distinguish between tangible and intangible entities. In other words, how to commemorate material artifacts in space that are, after launch, rather more virtual than tangible objects.

The bottom line is how to select, alongside the valuable sites, monuments, observatories and instruments, outstanding objects operating in space and how they could be listed as significant material artifacts among historically important astronomical facilities. Needless to say, Astronomical Space Observatories and Lunar and Planetary Space Vehicles have been of outstanding value to astronomy. Space observatories such as the Hubble Space Telescope, Quant, Chandra, WMAP, Spitzer, Planck, Kepler, etc. literally revolutionized our world attitude and tremendously advanced physics astrophysics and astronomy. In turn, planetary orbiters and landers such as Luna, Venera, Viking, Voyager, Galileo, Cassini-Huygens, Rosetta, etc opened up to our eyes neighbor space and allowed us to have close-up views of virtually all major members of the Solar system family.

The goal is therefore to find a consistent approach to the legacy of various human artifacts and activities in this particular field of astronomy which is intimately related with the progress of space technology. Mock-ups of several generations of world-recognized astronomical and planetary spacecraft preserved in space facilities around the world could serve as replicas of the virtual space objects and after acceptance by the international bodies (IAU, ICOMOS, etc.) could possibly be assigned as UNESCO Space World Heritage. In the short list of such objects one might suggest Yu. Gagarin's orbital flight capsule VOSTOK and Gagarin's space-suit; the Apollo 11 lander EAGLE and N. Armstrong's space-suit. Also in the list could be the first orbital stations Salyut, Skylab and MIR, which paved the way to the International Space Station (ISS), and several generations of space launchers including the Space Shuttle and Buran, as well as a few robotic spacecraft.

\section{Extraterrestrial Material/Lunar Soil and Proceedings/ Manuscripts of Space Pioneers}

These entities are of special importance in terms of Space World Heritage. Samples of extraterrestrial origin delivered by APOLLO astronauts and LUNA space vehicles back to Earth promoted unique opportunities to gain insight into the formation of the EarthMoon system and manifested the first steps undertaken by humans towards the in-depth study of pristine matter. They encapsulated unique information on the solar system's origin and early evolution. Currently, the main bulk of the samples are preserved in the Johnson Space Flight Center, Houston, USA, and in the Vernadsky Institute, Moscow, Russia. These samples represent real accomplishments of human culture and they deserve to be recognized as UNESCO World Heritage.

Among the important topics selected as space legacy, the Proceedings and/or Manuscripts of space pioneers could be considered. This particular aspect of Astronomical/Space 
World Heritage is addressed with a caveat to meet the criteria of the World Heritage Convention. Among space pioneers, one might suggest listing Konstantin Tsiolkovsky, Hermann Oberth, Robert Goddard, Yuri Kondratyuk, Serge Korolev, and Verner von Braun. The respective archives are pertinent to selecting necessary documents, publications, etc. in an appropriate format. There should be rather strict regulations while soliciting UNESCO patronage over these documents.

\section{Space Facilities and Launch Pads (Cosmodromes)}

This particular segment of technological heritage connected with space exploration must be specially addressed and could be considered as the first step towards the implementation of the new UNESCO Initiative. Indeed, Space Facilities where recognized spacecraft were designed and manufactured and Launch pads (cosmodromes) are regarded as an important part of the overall space infrastructure. They ensured the development and launch of spacecraft and thus are to be regarded as historical cornerstones of space exploration. Examples are: OKB-1 (RSC "Energiya"), NPO-Lavochkin, the Jet Propulsion Laboratory (JPL), Johnson Space Flight Center (JSFC), Toulouse Space Facility, Bayconour Space Center, Cape Canaveral Space Center, and Koru Launch Pad. One should bear in mind, however, that the existing formal restrictions are subject to rather elaborative negotiations before one could announce space ventures/cosmodromes as potential UNESCO World Heritage. Nonetheless, we are keen to anticipate solid progress towards understanding the principal concepts underlying such a complex topic which could be accomplished with a step-by-step approach.

Baykonour Launch pad could be suggested as a Case Study. Historically it was the first site linking mankind with the skies and it has a priority as the site holding the historical facilities that provided the launch of the first artificial satellite and the Gagarin flight, which manifested a great breakthrough in human civilization. It is one of the most advanced possessions of the space era and occupies a historically important position in human culture. Its recognized achievements and credibility would surely satisfy the Operational Guidelines of the World Heritage Convention (UNESCO 2015), which state in Article 49 that "Outstanding Universal Value means cultural and/or natural significance which is so exceptional as to transcend national boundaries and to be of common importance for present and future generations of all humanity". They also, surely, "represent a masterpiece of human creative genius" (ibid., Article 77(i)).

We are aware that the Case Study must satisfy the basic standards concerning the tangible evidence of sites and/or objects in order to be selected as cultural entities of globally recognized value. Unfortunately, until recently no special guidelines have been proposed by interested countries in order to satisfy the requirements and criteria for the selection of space science and technology sites/artifacts in order to fully accommodate UNESCO WH standards. Obviously, alongside the inventory and description of the historical development, management issues must be addressed as most critical in terms of the justification for inscription. They include comparative analysis, integrity-authenticity, criteria under which inscription might be proposed, suggested statement of OUV, etc., which are vital in the study.

Another important point one should bear in mind is that Baykonour is a huge area with numerous launch pads and facilities in its infrastructure; it has been dramatically extended since those historical events. Thus, in order to satisfy integrity-authenticity requirements, UNESCO patronage could be extended over only a small part of the overall Baykonour site/infrastructure - e.g., the famous "Launch pad No. 2" known as "Gagarin's start site". In addition to Gagarin's start site, two small cottages "Gagarin" 
and "Korolev", where the first cosmonaut and Chief Designer stayed overnight before the historical flight, could be suggested. Following the WH Convention (1972), such a selection is based upon tangible evidence. Gagarin's launch pad is in place and operational. The start's facility is integrated in terms of the capable space technologies used for the testing and launch of manned craft. Many cosmonauts and crews have been launched since the Gagarin flight. Also, in terms of integrity, it is important that no removal or partial demolition has occurred since Gagarin's time.

The authenticity is undoubted and well documented, including available historical archives. Commemoration of the historical events related to the launch pad is inscribed on the stone plate erected in situ beside the pad. While the original launch pad and close environment were partially reconstructed incorporating modern equipment this does not influence the authenticity. The Gagarin and Korolev cottages have been repaired but did not experience any changes: they include the former furniture and personal belongings. Thus Gagarin's start site and cottages satisfy the criteria under which inscription might be proposed, as well as contributing to the suggested statement of OUV. The places fully preserve the tangible attributes of their history and could be commemorated as UNESCO WH. This, it seems, will not impose political obstacles despite the fact that Baykonour is under international Russia-Kazakhstan joint jurisdiction. Some important issues are to be negotiated with ROSCOSMOS including managerial aspects of the implementation of the Initiative.

\section{Conclusion}

Space Astronomy and Planetary Exploration have ensured very significant progress in gaining knowledge about the Solar system and the Universe, which has tremendously broadened human horizons. There is a basic synergy between Space Astronomy and Space Technology, the latter serving a driver to progress with astronomy. It is therefore essential to include Space Astronomy/Technology, as represented by astronomical spacecraft and probes, as an important segment of Astronomical World Heritage. It is proposed to develop a consistent approach to the legacy of space facilities and human artifacts so that they could be recognized as Space Technology Heritage. Launch pads and groundbased space facilities could be selected as a starting point for accepting the proposed Space Heritage Initiative. The Initiative could be further expanded to other historically important achievements in space science and technology involving robotic and manned flights. An International Expert Working Group was set up under the UNESCO umbrella in 2012 to discuss the main issues and to develop proposals on how to progress the Space Technology Initiative and to accommodate the criteria of the World Heritage Convention.

\section{References}

Ruggles, C. \& Cotte, M. (eds.) 2010, Heritage Sites of Astronomy and Archaeoastronomy in the context of the UNESCO World Heritage Convention (Paris: ICOMOS -IAU)

UNESCO 2015, The Operational Guidelines for the Implementation of the World Heritage Convention (Paris: UNESCO) http://whc.unesco.org/en/guidelines 